REVIEW ARTICLE

\title{
Body fat and its influence on health and disease
}

\author{
P.A.J. Perera ${ }^{1}$ and E.M.I. Edirisinghe ${ }^{2, *}$ \\ ${ }^{1}$ Department of Biochemistry, Faculty of Medicine and Allied Sciences, Rajarata University of Sri Lanka, \\ Saliyapura 50008, Sri Lanka \\ ${ }^{2}$ Department of Food Science and Nutrition, Illinois Institute of Technology, Chicago, IL- 60616, USA
}

\begin{abstract}
This is a review of literature from 1963 taking into account the excellent reviews on body fat and its metabolism and scintillating research articles on the role of non-esterified fatty acid (NEFA) and body fat depots in maintenance of health and also their dysfunction leading to chronic diseases that affect quality of life. Main objective of this paper is to highlight the significance of elevated plasma NEFA in type 2 diabetes mellitus and obesity and the overall influence of depot fats in the maintenance of health. Reduction in receptor mediated cell function in muscle due to loss of insulin sensitivity arising from elevated non-esterified fatty acid is given due recognition and is considered as an alternate route for development of strategies for the control of chronic diseases associated with energy dysfunction. In addition, glyceroneogenesis in adipose tissues that accompany fat synthesis can also considered as a possible site which could be targeted to reduce the elevated plasma NEFA.
\end{abstract}

Keywords: Upper and lower body fat, gender differences, accretion of body fat, fat in pregnancy, plasma non esterified fatty acid.

\section{INTRODUCTION}

Obesity is becoming a gigantic problem both in the developed and developing world. It is closely associated with chronic diseases that affect quality and life span of human life. This association arises as a result of interaction of body fat depots on energy metabolism and consequences that follow owing to their derangement.

Body fat depots comprise of upper body fat, lower body fat and ectopic fat. Unlike the upper and lower body fat whose primary function is to provide energy to tissues during times of energy needs ectopic fat stores excess energy that spills over due to inability of the upper and lower body fat stores to handle it. This arises as a result of failure of subcutaneous adipose tissues to proliferate and differentiate leading to hypertrophy.

Ectopic fat depending on its location produce systemic or local effects. Systemic effects are shown by visceral, intrahepatic and intramuscular fat while local effects are shown by pericardial, renal sinus, myocardial steatosis and perivascular fat. These adipose tissues when triggered release adipokines resulting in inflammation and insulin resistance.

Increase in non-esterified fatty acid (NEFA) in plasma is well documented in obesity related type 2 diabetes and others associated with cardiovascular diseases. A reduction in its status is associated with increased insulin sensitivity. In the post-absorptive state, NEFA status in plasma is determined by its uptake into adipose tissues for storage, its use for metabolic functions of other tissues and its release from adipose tissues by lipolysis. Laying down of fat in adipose tissues is determined by uptake of NEFA and its esterification with glycerol while its release is dependent on lipolysis. These are two juxtapositions in fat metabolism that are worthwhile to be considered for lowering NEFA in blood plasma. Fatty acid release from stores will depend on their metabolic activity; upper body fat will mobilize fatty acid faster than lower body fat.

Action is needed to reduce upper body fat, by way of its mobilization and deposition. The former could be accomplished by aerobic type oxidation and the latter by reduction of dietary fat intake, especially saturated fat. Along with these theoretical considerations, emphasis of a normal body mass index is of prime importance. 


\section{FAT DEPOTS}

Body fat is stored in adipose tissues distributed in different parts of the body. Fat depots in these adipose tissues vary in respect of fat storage, fat mobilization and function. The fat deposits can be broadly divided into upper body fat and lower body fat. The upper body fat comprises of fat stored in abdominal subcutaneous and visceral adipose tissues while lower body fat comprises of fat stored in gluteo-femoral adipose tissues (Karpe and Pinnick, 2015).

\section{Upper and lower body fat}

Upper body fat (UBF) has a high lipid turnover and demonstrates a strong lipolytic response to stress hormones. Fat deposition at this site takes place mostly via chylomicrons. Lower body fat with a low lipid turnover has fat deposited in its depot mostly via VLDL made available through the redistribution pathway (Karpe and Pinnick, 2015).

UBF is laid down as large fat droplets through adipocyte hypertrophy whilst lower body fat is laid down as small fat droplets through adipocyte hyperplasia. There is a constant flux of free fatty acids continuously entering and leaving fat depots. The net direction of the flux is controlled by insulin and leptin, both increasing inward flux of fatty acids while outflow of fatty acids is controlled through a balance between lipolytic beta adrenergic receptors and anti-lipolytic alpha 2 receptors (Tchoukkalova et al., 2008).

Reduction of dietary energy accompanies a reduction in the abdominal upper body subcutaneous fat and not gluteo-femoral adipose tissue fat (Kopelman, 1997). The latter is the one which is used prominently during lactation in the post-absorptive state by women, a mechanism available to lose some of the fat gained during pregnancy (McFarland, 1997).

The divergent functional differences cited above between UBF and LBF do suggest likely presence of site specific sets of different developmental genes under epigenetic control. The observation that regional characteristics are retained when tested in vitro indicates that adipocytes heterogeneity is inherited rather than consequence of local micro environment variation (Karpe and Pinnick, 2015). Multiple studies have examined transcriptional profile of different adipose tissues and identified marked depot specific differences in the expression of developmental transcription factors (Karastergiou et al., 2013). Depot specific differences are seen between apparently similar upper body subcutaneous and lower body subcutaneous depots. When health aspects of body fat distribution is looked into, LBF is found to exhibit a protective role showing opposing association with risk of cardiovascular disease and type 2 diabetes mellitus whereas the upper body fat is seen to have the opposite effect (Karpe and Pinnick, 2015).

Considering the importance of body fat distribution in determining 'metabolic health' and opposing associations observed between metabolic risk factors and upper and lower body fat there is a need to investigate anthropometric indices that reflect upper and lower body fat in relation to health and diseases (Pouliot et al., 1994).

\section{Ectopic fat depots}

This refers to excess adipose tissue in locations traditionally not associated with energy storage. It includes intra-abdominal visceral adipose tissue, a characteristic of upper body obesity. One hypothesis is that in states of positive energy balance when capacity of subcutaneous tissue (SAT) is reached storage shifts to ectopic sites, including viscera, heart, and vasculature. The failure of SAT to store additional non-esterified fatty acid (NEFA) is believed to result from a failure of proliferation and differentiation of adipocytes leading to hypertrophy (Despres et al.,2008; Heilbron et al., 2004).

Fat stored in ectopic depots depending on their location may produce systemic or local effects. Those having potential systemic effects include visceral adipose tissue (VAT) fat, intrahepatic fat (fatty liver) and intramuscular fat while those having potential local effects include pericardial (pericoronary) fat, renal sinus fat, myocardial steatosis and perivascular fat.

Consistent with this theory, the degree of subcutaneous abdominal adipose cell hypertrophy has been shown to predict the development of type 2 diabetes mellitus. In addition, thiazolidinediones, which improve insulin sensitivity, have been shown to promote differentiation of new fat cells in subcutaneous fat without an increase in visceral fat. Taken together, these data suggest that ectopic fat 
deposition may result from the failure of SAT to act as a metabolic sink (Britton and Fox, 2011) and that its accumulation may put a person at risk in developing diabetes type 2 .

With development of obesity, VAT becomes infiltrated with macrophages followed by upregulation of adipokines. These adipokines play a role in the development of inflammation and insulin resistance. Dysregulated production of adipokines due to adipose dysfunction can contribute towards obesity linked complications (Ouchi et al., 2011).

\section{FAT SYNTHESIS IN ADIPOSE TISSUE}

Fat is synthesized using NEFA delivered by plasma chylomicrons and VLDL and glycerol synthesized from glucose delivered by plasma. It is to be noted that glucose taken up by adipose tissues is not used for fatty acid synthesis but wholly or partly responsible for providing the glycerol skeleton required for triacylglycerol synthesis (Nye, Kim et al., 2008). If the uptake of glucose and glyceroneogenesis in adipose tissue are affected it may result in elevated NEFA. Thus, upregulation of glyceroneogenesis may be an alternative method of taking care of increased NEFA in plasma. It has been estimated that concentration of NEFA in the blood of an obese human after an overnight fast $(0.8 \mathrm{mM})$ is 3 - 4 times that of a non-obese individual $(0.2$ $\mathrm{mM}$ ) and in individuals with type 2 diabetes the level could rise to as high as $3.3 \mathrm{mM}$ (Roden, 2004).

\section{GENDER DIFFERENCES}

There is a gender difference in total body fat and its distribution. Women have a higher percentage of fat than men. Men tend to accumulate body fat intra abdominally giving the body an 'apple' shape while women accumulate fat in the gluteal femoral region giving the body a 'pear' shape (Karastergiou et al., 2012). There are distinct regional differences in the metabolism of fatty acids between men and women (Blaak, 2001). There is in vivo evidence to show that catecholamine mediated leg free fatty acid release is lower in men than women. Adipocytes in abdominal fat tissue differ from those found in the gluteal femoral region. Abdominal fat has a preponderance of beta receptors, whereas alpha receptors predominate in the gluteal fat. Alpha receptors promote accumulation of fat within cells whereas beta receptors favor mobilization of fat stores.

\section{MODULATION OF FAT DISTRIBUTION BY SEX STEROIDS}

Characteristic sex differences arise at puberty. Boys increase their body weight mainly by increase of lean body mass while girls increase their body weight mainly by increase of fat mass. Typical android and gynoid fat distribution of fat takes place during this period. In women, fat is centrally redistributed in menopause probably following a fall in estrogen level. In men, there is an increase in visceral adiposity in old age following a fall in testosterone level. Transgender men and women show clear shifts in fat distribution following treatment with sex steroids (Karastergiou et al., 2012).

\section{Atherogenesis}

Epidemiological studies have shown that smaller LDL particles are more atherogenic than larger LDL particles (Carmena et al., 2004; Gardner et $a l$. , 1996) and women produce larger LDL particles compared to men (Nikkila et al., 1996). This may be an important factor that makes women less prone to develop cardiovascular diseases (CVD), arising from the reduced opportunities that large LDL particles provide when subjected to oxidation by oxygen free radicals (Gardner et al., 1996). Among diabetics of type 2 visceral adipose tissue was negatively related ( $\mathrm{P}<0.0001)$ to LDL size (Sam et al., 2008).

LDL particles laden with lipids are rich in cholesterol. An increase in the LDL particle size corresponds to a relative increase in the cholesterol concentration compared to other lipoprotein constituents that are the targets of oxidation by free radicals. LDL particles are the main suppliers of cholesterol to rapidly dividing cells and tissues synthesizing steroidal hormones. Their uptake is receptor mediated and dependent on the presence of apo-B receptors. If their stay in the blood is prolonged, it could lead to higher risk of oxidation by oxygen free radicals. This could result in lowered apo B receptor mediated uptake of oxidized LDL particles initiating the process of atherosclerosis (Lamarche et al., 1997). It is to be noted that it is the protein and phospholipid containing unsaturated fatty acid on the surface of the lipoprotein that are prone to oxidation and not cholesterol which is robust. In order to dispose cholesterol from the body as bile acid, an initiation step 7 - $\alpha$-hydroxylase is mandatory indicating that human does not have 
the ability to oxidize cholesterol (Jelinek et al., 1990) While appreciating that female reproductive hormones and the cyclical nature of their variation in blood plasma enable blood to maintain blood lipids at a lower threshold level, the likely mechanism that confers some type of safety against CVD may be related to the manner in which lipoproteins are handled by the body. Serum HDL cholesterol level of women of the reproductive age group is significantly higher than those of men of comparable age and status. The inbuilt safety factors such as high protein content and antioxidant status of HDL do confer solubility and stability to the lipoprotein compared to all others in circulation. It is to be noted that HDL status does increase in pregnancy providing further safety towards a productive outcome of pregnant women. HDL is known to confer protection by offering scavenging facilities to circulating cholesterol, transport facilities to oxidized LDL particles, and opposing the action of oxygen free radicals (Soran et al., 2015; Huang et al., 2013).

\section{ENDOTHELIAL FUNCTIONS AND FAT}

Research done over the last two decades suggests that vascular endothelial dysfunction may contribute to the pathophysiology of hypertension, clinical ischemia, myocardial infarction, and sudden death (Paterick and Fletcher, 2001). Endothelial functions include vasomotor tone, constriction and dilation, balance between fibrinolysis and thrombosis, control of the inflammatory response, and growth of vascular smooth muscle. Effects of lipids have been documented on many of the endothelial processes (Davignon and Ganz, 2004; Shimokawa, 1999). In particular, there is strong evidence to suggest that the circulatory blood lipids affect vasodilatation mediated by endothelium derived nitric oxide with the interaction between leukocytes and the endothelial surface. Vasodilatory mechanism is an important feature in regulation of normal blood pressure. LDL cholesterol, triglyceride rich lipoproteins and free fatty acids impair endothelium dependent vasodilatation. The effects of lipids on endothelium dependent vasodilatation can be reversed not only by reducing the level of elevated blood lipids levels, but also by providing dietary antioxidants, suggesting a mechanism related to oxidative stress (Trpkovic et al., 2015).

\section{ACCRETION OF BODY FAT IN THE YOUNG}

Generally, after 25 years weight gain mainly results from body fat accumulation. Men have $20 \%$ body fat at 25 years, $30 \%$ at 55 years and $35 \%$ at 75 years (Gropper and Smith, 2013). In addition to fat gain lean body mass decreases due to a reduction in protein and body cell mass synthesis. More marked increases in fat deposits occur in the females with increase in age.

Of the different forms of fat, essential fat $(\mathrm{EF})$ is necessary to maintain life and reproductive functions. This fat includes fat associated with bone marrow, central nervous system, internal organs and cell membranes. The percentage of essential body fat for women is greater than that for men, due to additional reproductive functions. The percentage of essential fat for men is $3-5 \%$ and for women is $8-12 \%$ of body weight.

Part of body fat stored in adipose tissue protects internal organs in the chest and abdomen. The minimum recommended total body fat percentage exceeds the essential fat percentage value (Gropper and Smith, 2013).

Table 1: The average fat characteristics of reference man of body weight $70 \mathrm{~kg}$ and reference woman of body weight 56.8 $\mathrm{kg}$ of age 20-24 years.

\begin{tabular}{llll}
\hline & Total body fat & Storage fat & Essential fat \\
\hline Man $(\mathrm{kg})$ & $10.5(15.0 \%)$ & $8.4(12 \%)$ & $2.1(3 \%)$ \\
Woman $(\mathrm{kg})$ & $15.4(27.0 \%)$ & $8.5(15.0 \%)$ & $6.8(12 \%)$ \\
\hline
\end{tabular}

(Source: Gropper and Smith, 2013). 


\section{Age related fat depot changes}

Fat is redistributed among different fat depots over time, especially during and after middle age fat redistributes from subcutaneous to intraabdominal visceral depots (Meunier et al., 1971). In old age, fat is redistributed outside fat depots, accumulating in bone marrow, muscle, liver and other ectopic sites. Presence of excess dysfunctional depot fat appears to accelerate onset of multiple age related diseases. These include diabetes, hypertension, cancer, cognitive dysfunction, and atherosclerosis leading to heart attacks and stroke. Experiments with animals have shown that ablation of excess dysfunctional fat and mutations affecting such fat do enhance health span and maximum life span (Tchkonia et al., 2010).

\section{Pregnancy related fat and fetal development}

For a productive pregnancy, there is a demand for body fat. An average value of $20 \%$ has been proposed, but sufficient research has not been done to validate it. Women who are obese and sports women in peak form with very little body fat are unable to conceive. The reasons for this are unclear.

Pregnancy is characterized by a series of metabolic changes that caters to the demands of the growing fetus and the supportive tissues of the pregnant woman that carries it. After parturition a fat store of $4 \mathrm{~kg}$ on the average (WHO, 1985) is left partly to meet the energy requirements of the newborn and the mother. This fat, mostly of femoral-gluteal origin is used for milk fat synthesis during lactation (McFarland, 1997).

An important aspect of pregnancy is early accretion of adipose tissue followed by development of insulin resistance facilitating lipolysis in late pregnancy. Insulin secretion increases in early pregnancy without change in insulin sensitivity. However, in late pregnancy fat deposit in maternal adipose tissue declines while postprandial non-essential fatty acid (NEFA) levels increase. In pregnancy, insulin mediated glucose disposal decreases by nearly $50 \%$ when compared with pre-pregnancy status. Ability of insulin to suppress lipolysis is reduced. This result in increased blood glucose and NEFA levels to support of fetal growth and prepare maternal tissues for parturition and nourishment of the newborn (Barbour et al., 2007). This does indicate that plasma NEFA has a role in directing appropriate energy sources via suppression of insulin activity from the very beginning of life.

Placental hormones secreted in late pregnancy stages have been suggested to impair insulin mediated handling of glucose by maternal skeletal muscle and availability of increased glucose for fetal growth. However, changes in placental hormones do not correlate with changes in maternal insulin resistance (Kirwan et al., 2002). It is to be noted that just as much placental hormones have been implicated in the reduction of insulin sensitivity, the presence of increased NEFA level too need to be taken into account as it is also being increasingly recognized as a desensitizing factor.

In gestational diabetes, this situation is further aggravated by the presence of greater postprandial increase in NEFA, increased hepatic gluconeogenesis and severe insulin resistance. It needs to be emphasized that increased NEFA is intimately related to decrease in insulin sensitivity especially with reference to uptake of glucose into muscle and adipose tissues which are considered insulin dependent tissues (Barbour et al., 2007)

\section{Milk fat etiology}

Breast milk is continuously synthesized to cater to the needs of the newborn. After a meal, chylomicrons absorbed by the mother provide fatty acid required for the synthesis of milk fat while glycerol is derived from glucose in her blood. Thereafter in the post-absorptive state the mother preferentially uses lower body fat stored in gluteo-femoral adipose tissues (McFarland, 1997)

\section{FATTY ACID, INSULIN RESISTANCE AND ASSOCIATED METABOLIC DISORDERS}

Of the fuel molecules, fatty acid is the principal energy supplier and its derivative fat the principal energy store. There is constant interchange between these two sources via blood. An elevation in the level of NEFA in the blood is known to inhibit the uptake of glucose into muscle, a property associated with insulin dysfunction. Resistance to insulin results in excessive flux of fatty acid as a result of unopposed adipose tissue lipolysis. Accumulation of NEFA can further increase insulin resistance by modulating insulin receptor expression and post-receptor signaling. 
Two of the most common clinical syndromes associated with insulin resistance are type 2 diabetes and metabolic syndrome. Others include cardiovascular diseases, essential hypertension, polycystic ovary syndrome, nonalcoholic fatty liver disease, sleep apnoea and certain form of cancer.

\section{OBESITY}

Insulin resistance has been associated with obesity. Lipid induced insulin resistance in skeletal muscle is reported to stem from defects in insulin stimulated glucose transport. The steatotic liver is also resistant to insulin as per inhibition of hepatic glucose production and stimulation of glycogen synthesis. In muscle and liver, intracellular accumulation of lipids trigger activation of protein kinase $\mathrm{C}$ with impairment of insulin signaling (Samuel et al., 2010)

Elevated NEFA concentrations in obesity that directly induce inflammatory responses in adipocytes have been proposed as one of the mechanisms associated with insulin resistance (Boden, 2011). NEFA activate NF-kB transcription factor and cytokine production in adipocytes. In particular, saturated fatty acids activate macrophage like-cells via the Toll-like receptor (TLR4). TLR4 on adipocytes acting as sensor of elevated NEFA concentrations initiates NF- k B mediated signaling cascade creating an inflammatory environment resulting in insulindesensitizing processes. NEFA also induces oxidative stress influencing insulin signaling as well as insulin receptor expression (Kokoeva et al., 2006).

Most of the obesity related depot fat is truncal and comprises of subcutaneous upper body fat. It is related to a reduction in adipocyte derived hormone adiponectin in plasma. When measures are taken to reduce obesity, it is found that the plasma adiponectin level is increased. It is negatively correlated with body fat and opposes insulin resistance.

In terms of overall health, lower body subcutaneous fat in thighs and buttocks has some potential benefits. But upper body deeper visceral fat puts the person at risk from cardiovascular disease. There is emerging evidence to indicate that protruding big bellies and large waist are signs of visceral fat. Visceral fat is in a gel form and wraps around major organs, including liver, pancreas and kidneys. It is pronounced in obese people. Many can have visceral fat without knowing it. Excess visceral fat is linked to increased risk of obesity, diabetes, coronary heart disease, stroke, sexual dysfunction, arthritis, dementia, sleep disorders and cancer. .

Waist circumference and abdominal sagittal diameter are simple anthropometric indices of abdominal visceral adipose tissue and are related to cardiovascular risk in men and women (Pouliot et al., 1994).

Visceral fat is considered toxic and is said to provoke inflammatory pathways and produce signaling molecules that can interfere with body's normal hormonal function. Indeed, obesity has been recognized as chronic lowgrade inflammatory status in the body. The primary cause for obesity-induced inflammation is not yet fully understood. However, it has been proposed that initiation of inflammation in obesity is related to endoplasmic reticulum (ER) stress. Over nutrition causes ER stress in liver and adipose tissue due to excess lipid accumulation and energy metabolism. ER stress initiates a stress response signaling cascade by activating IKK, the MAPKs p38 and JNK, and finally the major inflammatory transcription factor NF- k B. Consequently, adipose tissue produces large amounts of inflammatory cytokines and chemokines, collectively called adipokines, such as TNF, IL- 1, IL-6, and MCP-1 (Hummasti and Hotamisligil., 2010; Ozcan et al., 2004). Cytokines are also known to interfere with hormones that regulate appetite, weight, mood and brain function.

\section{DIABETES}

Resistance to insulin in the presence of excessive flux of fatty acid as a result of unopposed adipose tissue lipolysis is well known. Insulin resistance plays a central role in the etiology of type 2 diabetes that effects insulin action on glucose uptake. The association of obesity with increased risk of developing type 2 diabetes has been recognized for decades, and the major link is the ability of obesity to engender insulin resistance (Kokoeva et al., 2006).

Insulin is a critical regulator in all aspects of adipocyte biology. Insulin promotes adipocyte triglyceride stores by a number of mechanisms, including promoting the differentiation of preadipocytes to adipocytes ((Kokoeva et al., 2006; Zeyda and Stulnig, 2009). In mature 
adipocytes, insulin stimulates glucose transport and lipogenesis, as well as inhibiting lipolysis especially in fed state. Further, insulin promotes synthesis of triacylglycerol from fatty acid delivered to adipocytes via chylomicrons and VLDL and glycerol derived from glucose via glycolysis. In the fed state, insulin induces dephosphorylation of hormone sensitive lipase by reducing cAMP and activating phosphoprotein phosphatase. This inhibits lipase activity and decreases fat hydrolysis. In the fasting state glucagon and epinephrine mobilize stored fat via hormone sensitive lipase releasing fatty acid into blood.

In 1963, Randle et al. ascribed elevated NEFA to be the likely causative factor for impaired carbohydrate tolerance and decreased insulin sensitivity. They proposed the term "glucose fatty acid cycle" to denote interactions between glucose and fatty acid metabolism in peripheral tissues for control of blood glucose and fatty acid levels. Following this finding, Schalch and Kipnis (1965) reported development of impaired carbohydrate tolerance and decreased sensitivity to insulin with elevated fasting plasma NEFA levels in pregnancy, obesity, maturity-onset diabetes, acromegaly, and in subjects given exogenous growth hormone.

Guenther Boden (2011) hypothesized that elevated plasma NFFA levels were to a large part a cause for insulin resistance in obese patients with type 2 diabetes. Insulin resistance is now given special recognition because of its close association with type 2 diabetes, hypertension, dyslipidemia, and abnormalities in blood coagulation and fibrinolysis and all of which are considered independent risk factors for cardiovascular disease (Bray, 2004). Does this mean that fatty acid and its receptors (Ichimura et al., 2014) can be considered a modulator of insulin activity and therefore be eligible to be considered as a target for development of dietary and alternate pharmacological interventions in the future?

\section{METABOLIC SYNDROME}

In 1988, it was proposed that individuals with glucose intolerance, elevated triacylglycerol, low HDL cholesterol and essential hypertension were at great risk in developing cardiovascular disease. It was referred to as Syndrome $\mathrm{X}$ and later changed to Metabolic Syndrome. There is close relation between increased fatty acid flux and metabolic syndrome which is recognized by a group of risk factors that multiply a person's risk for heart disease, diabetes and stroke (Grundy et al., 2004).

With incidence of type 2 diabetes and obesity on the rise in both developed and developing countries, it is time that all countries took cognizance of all possible solutions to this problem and took preventive actions to curtail it.

In alleviating the above situations, one serious theoretical consideration that requires further investigation is to understand the role played by NEFA especially via receptors in regulation of body energy metabolism and the influence of differently placed fat depots in controlling the level of NEFA and their ultimate effect on the quality of life (Ichimura et al., 2014).

\section{REFERENCES}

Barbour, L.A., McCurdy, C.E., Hernandaz, T.L., Kirwan, J.P., Catalano, P.M. and Friedman, J.E. (2007). Cellular mechanisms for insulin resistance in normal pregnancy and gestational diabetes. Diabetes Care. 30 (2): S112-S119.

Blaak, E.E. (2001). Gender differences in fat metabolism. Current Opinion in Clinical Nutrition Metabolism Care. 4 (6): 499-502.

Boden, G. (2011). Obesity, insulin resistance and free fatty acids. Current Opinion in Endocrinology, Diabetes and Obesity. 18 (2): 139-143.

Bray, G.A. (2004). Medical consequences of obesity. Journal of Clinical Endocrinology Metabolism. 89: 2583-2589.

Britton, K.A. and Fox, C.S. (2011). Ectopic fat depots and cardiovascular disease. Circulation. 124: 837841.

Davignon, J. and Ganz, P. (2004). Role of endothelial dysfunction in atherosclerosis. Circulation. 109 (23/1): 27-32.

Despres, J-P., Lemiuex, I., Bergeron, J., Pibarot, P., Mathieu, P., Larose, E., Rodes-Cabau, J., Bertrand, O.F. and Poirier, P. (2008). Abdominal obesity and the metabolic syndrome: contribution to global cardiometabolic risk. Arteriorsclerosis Thrombosis Vascular Biology. 28: 1039-1049.

Gardner, C.D., Fortmann S.P. and Kraus, R.M. (1996). Association of small low-density lipoprotein particles with the incidence of coronary artery disease in men and women. Journal of American Medical Association. 276 (11): 875-881.

Gropper, S.S. and Smith, J.L. (2013). Changes in body mass with age. Advanced Nutrition and 
Human Metabolism. Sixth edition. Wadsworth. Delmont, California Pp. 237-238.

Grundy, S.M., Brewer, H.B. Jr., Cleeman, J.I., Smith, S.C. Jr., and Lenfant, C. (2004). Definition of metabolic syndrome. Circulation. 109 (3): 433438.

Heilbron, L., Smith, S.R. and Ravussin, E. (2004). Failure of fat cell proliferation, mitochondrial function and fat oxidation results in ectopic fat storage, insulin resistance and type II diabetes mellitus. International Journal of Obesity Related Metabolism Disorders. 28 (4): 12-21.

Huang, Y., Wu, Z., Riwanto, M., Gao, S., Levision, B.S., Gu X., Ichimura, A., Hasegawa, S., Kasubuchi, M. and Kimura, I. (2014). Free fatty acid receptors as therapeutic targets for the treatment of diabetes. Frontiers in Pharmacology. 5: 236. Published on line 2014 Nov 6 . PMCID:PMC4222138

Hummasti, S. and Hotamisligil, G. S. (2010). Endoplasmic reticulum stress and inflammation in obesity and diabetes. Circulation Research 107: 579-591

Karastergiou, K., Fried, S.K., Xie, H., Lee, M.J., Divoux, A., Rosencrantz, M.A., Chang, R.J. and Smith, S.R. (2013). Distinct developmental signatures of human abdominal and gluteal subcutaneous adipose tissue depots. Journal of Clinical Endocrinology Metabolism 98 (1): 362371

Karastergiou, K., Smith, S.R., Greenberg, A.S. and Fried, S.K. (2012). Sex differences in human adipose tissues - the biology of pear shape. Biology of Sex Differences 3: 13.

Karpe, F. and Pinnick, K.E. (2015). Biology of upperbody and lower-body adipose tissue - link to whole body phenotypes. Nature Reviews Endocrinology 11: 90-100.

Kirwan, J.P., Hauguel, D.M.S., Lepercq, J., Challier, J.C., Huston, P.L., Friedman, J.E., Kalhan, S.C. and Catalano, P.M. (2002). TNF-alpha is a predictor of insulin resistance in human pregnancy. Diabetes 51: 2207 - 2213.

Lamarche, B., Tchernof, A., Moorjani, S., Cantin, B., Dagenaise, G.R., Lupien, P.J. and Despres, J.P. (1997). Small, dense low-density lipoprotein particles as a predictor of the ischemic heart disease in men. Prospective results from the Quebec cardiovascular study. Circulation. 95: 69 75.

McFarland, R. (1997). Female primates: fat or fit? In: M.E. Morbeck, A. Galloway and A.L. Zilhman (Eds.), The Evolving female, Princeton University Press, Princeton Pp. 163 -175.

Nye, C., Kim, J., Kalhan, S.C., Hanson, R.W. (2008). Reassessing triglyceride synthesis in adopose tissue. Trends in Endocrinology and Metabolism. 19 (10):356 - 361.

Nikkila, M., Pitkajarvi, T., Koivula, Solakivi, T., Lehtimaki, T., Laippala, P., Jokela, H., Lehtomaki,
E., Seppa, K. and Sillanaukee, P. (1996). Atherosclerosis. Women have larger and less atherogenic low density lipoprotein particle size than men. 119 (2): 181-190.

Ouchi, N., Parker, J.L., Lugus, J.J. and Walsh, K. (2011). Adipokines in inflammation and metabolic disease. Nature Reviews Immunology. 11 (2): 85 - 97.

Ozcan, U., Cao, Q., Yilmaz, E., Lee, A.H., Iwakoshi, N.N., Ozdelen, E., Tuncman, G., Gorgun, C., Glimcher, L.H. and Hotamisligil, G.S. (2004). Endoplasmic reticulum stress links - obesity, insulin action, and type 2 diabetes. Science. 306: 457-461.

Patel, S. R., Hayes, A. L., Blackwell, T., Evans, D. S., Anconi-Israel, S., Wing, Y. K. and Stone, K. L. (2014). The association between sleep and obesity in older adults. International Journal of Obesity. 38 (9); 1159-1164.

Paterick, T.E. and Fletcher, G.F. (2001). Endothelial function and cardiovascular prevention: role of blood lipids, exercise, and other risk factors. Cardiology Reviews. 9 (5): 282-286.

Pouliot, M.C., Despres, J. P., Liemieux, S., Moorjani, S., Bouchard, C., Tremblay, A., Nadeau, A. and Lupien, P.J. (1994). Waist circumference and abdominal sagittal diameter. Best simple anthropometric indexes of abdominal visceral adipose tissue accumulation and related cardiovascular risk in men and women. American Journal of Cardiology. 73: 460-468.

Randle, P.J., Garland, P.B., Hales, C.N. and Newsholm, E.A. (1963). Glucose fatty acid cycle. Its role in insulin sensitivity and the metabolic disturbances in diabetes mellitus. Lancet. 1: 785789.

Roden, M. (2004). How free fatty acids inhibit glucose utilization in human skeletal muscle. News Physiological Science. 19: 92 -96.

Samuel, V.T., Peterson, K.F. and Shulman, G.I. (2010). Lipid induced insulin resistance: unraveling the mechanism. Lancet. 375 (9733): 2267-2277.

Schalch, D.S. and Kipnis, D.M. (1965). Abnormalities in carbohydrate tolerance associated with elevated plasma non-esterified fatty acids. Journal of Clinical Investigation. 44 (12): 2010-2020.

Shi, H., Kokoeva, M.V., Inouye, K., Tzameli, I., Yin, H. and Flier, J.S. (2006). TLR4 links innate immunity and fatty acid-induced insulin resistance. Journal of Clinical Investigation. 116: 3015-3025.

Shimokawa, H. (1999). Primary endothelial dysfunction: atherosclerosis. Journal of Molecular Cell Cardiology. 31 (1): 23-37.

Tchkonia, T., Morbeck, D.E., Zglinicki, T.V., Deursen, J.V., Lustgarten,J., Scrable, H., Khosla, S., Jensen, M.D. and Kirkland, J.L. (2010). Fat 
tissue, aging and cellular senescence. Aging Cell. 9: 667-684.

Tchoukkalova, Y.D., Koutsari, C., Karpyak, M.V., Votruba, S.B., Wedndland, E. and Jensen, M.D. (2008). Subcutaneous adipocytes size and body fat distribution. American Journal of Clinical Nutrition. 87: 56-63.

Trpkovic, A., Resanovic, I., Stanimirovic, J., Radak, D., Mousa, S.A., Cenic-Milosevic, D., Jevremovic, D. and Isenovic, E.R. (2015).
Oxidized low-density lipoprotein as a biomarker of cardiovascular diseases. Critical Reviews in Clinical Laboratory Science. 52 (2):70-85.

World Health Organization (1985). Requirements during lactation. Technical Report on Energy and Protein Requirements. 724. Pp 87-88.

Zeyda, M. and Stulnig, T.M. (2009). Obesity, inflammation, and insulin resistance - a minireview. Gerontology. 55 (4): 379-86. 
